\title{
Spectroscopic studies of Lewis acid-base complexes-I
}

\section{Vibrational spectra and assignments for trimethylymine complexes of boron halides*}

\author{
Robert L. Amster $\dagger$ and Robert C. TAylor \\ Department of Chemistry, The University of Michigan, Ann Arbor, Michigan
}

(Received 13 January 1964)

\begin{abstract}
Infrared and Raman spectra of 1:1 complexes of trimethylamine with boron trifluoride, boron trichloride and boron tribromide have been obtained in the solid state. Samples studied included those isotopically enriched in $B^{10}$ as well as those containing the natural isotopic ratio of $B^{11}$ to $B^{10}$. On the basis of isotopic shifts and trends of frequencies within the series, vibrational assignments of the observed frequencies are proposed.
\end{abstract}

\section{INTRODUCTION}

LEWIS acid-base complexes have been of considerable chemical interest for many years. Not only do they provide examples in which chemical reaction and bond formation may occur without the usual complications of an initial bond rupture, but also they frequently play an important role in the reactions of the very interesting compounds of the third group elements. Spectroseopic studies, which can be expected to contribute greatly to the specific information about these compounds as well as to their broader aspects, have not been extensive. The continuing interest and importance of these compounds to inorganic chemistry and to catalytic processes warrant a greater degree of spectroscopic study than has yet appeared.

Trimethylamine (TMA) is well known as one of the stronger Lewis bases while the boron halides are recognized as typical Lewis acids. The well characterized $1: 1$ solid molecular complexes which are formed when trimethylamine reacts with $\mathrm{BF}_{3}[1-3]$, $\mathrm{BCl}_{3}[4-6]$ or $\mathrm{BBr}_{3}[7]$ provide an excellent series of compounds for investigation. The $\mathrm{BI}_{3}$ complex apparently has not been reported. $\mathrm{X}$-ray diffraction work on the boron trifluoride complex [8] has shown that this molecule possesses $\mathrm{C}_{3 v}$ symmetry with a dative bond between the boron and nitrogen atoms. The $\mathrm{BF}_{3}$ group, which is planar in the free state, is distorted into a pyramid in the complex with smaller

* Based on a dissertation submitted by Roвert L. Amster in partial fulfillment of the requirements of the Ph.D. degree to the Horace H. Rackham School of Graduate Studies, The University of Michigan, June 1961.

$\dagger$ Present address: Department of Chemistry, The University, Sheffield, England.

[1] J. R. Bright and W. C. Fernelius, J. Am. Chem. Soc. 65, 735 (1943).

[2] A. B. Burg and A. A. Green, $J$. Am. Chem. Soc. 65, 1838 (1943).

[3] G. M. Phulits, J. S. Hunter and L. E. Sutton, J. Chem. Soc. 146 (1945).

[4] D. R. Martin, Chem. Rev. 34, 461 (1944).

[5] W. Germatid and M. F. Latpert, Chem. Rev. 68, 1081 (1958).

[6] D. T. Hurd and R. C. Osthoff, Inorganic Syntheses, vol. 5, McGraw-Hill, New York (1957).

[7] R. C. Osthoff, C. A. Brown and F. H. Clarke, J. Am. Chem. Soc. 73, 4045 (1951).

[8] S. Geller and J. L. HoArd, Acta Cryst. 4, 399 (1951). 
changes occurring in the amine half of the molecule. The structures of the TMA complexes with the other two boron halides are presumed to be similar to that of the fluoride although no data are available.

Infrared frequencies of the boron trifluoride complex as a solid and in solution have been reported in two papers. $[9,10]$ and in an unpublished report [11]. Vibrational assignments based on these data have been proposed but points of disagreement exist. No Raman results have been located for this compound and the infrared data are restricted to the rock salt region.

Both Raman and infrared studies have been published for trimethylamine-boron trichloride [9-12]. Katritzky, and Peterson and Bauer discussed assignments in some detail and the latter workers carried out force constant calculations based on their assignments. The only Raman work is that by GoubEaU and co-workers [12] who reported frequencies only. Their results disagree with the infrared data.

Infrared studies of the trimethylamine-boron tribromide complex $[7,10]$ are quite incomplete and limited to the rock salt region. Only a few assignments have been suggested and Raman work does not appear to have been done. No spectral results for any of the complexes in which the boron was isotopically enriched in $\mathrm{B}^{10}$ have been found.

The incompleteness of the experimental data and the fragmentary and conflicting vibrational assignments for these molecules make a thorough spectroscopic study of the series desirable.

\section{EXPERIMENTAI}

\section{Reagents}

Boron trichloride and boron tribromide containing the nalural 4:1 isolopic abundance of $\mathrm{B}^{11}$ to $\mathrm{B}^{10}$ were obtained from commercial sources and used without other purification than a preliminary trap to trap distillation. A commercial sample of the diethyl etherate of boron trifluoride was vacuum distilled to give a clear liquid and was not purified further.

Isotopically enriched boron containing 92 per cent $\mathrm{B}^{10}$ was obtained from the Atomic Energy Commission in the form of $\mathrm{KBF}_{4}$. This was decomposed under vacuum at $750^{\circ} \mathrm{C}$ to give boron trifluoride. Boron- 10 enriched $\mathrm{BCl}_{3}$ and $\mathrm{BBr}_{3}$ were prepared from $\mathrm{BF}_{3}$ by the halogen exchange method described by GambLE [13]. A small amount of $\mathrm{KBF}_{4}$ containing 99 per cent boron-11 was obtained from the Oak Ridge National Laboratory and was used in the preparation of one sample of the $\mathrm{BF}_{3}$ complex. Anhydrous trimethylamine was purified by allowing it to stand over $\mathrm{P}_{2} \mathrm{O}_{5}$ at $-78^{\circ} \mathrm{C}$ to remove traces of water and of mono- and dimethylamines.

\section{Preparation of complexes}

The trimethylamine-boron trifluoride complexes were prepared by bubbling trimethylamine vapor through an icc-cold solution of dicthyl cther boron trifluoride

[9] H. J. Coenver and C. Currax, J. Am. Chem. Soc. 80, 3522 (1958).

[10] A. R. Katritzky, J. Chem. Soc. 2049 (1959).

[11] C. C. Peterson and S. H. BAuer, The Infrared Spectra of Lewis Salts, N.R.L. Report, Department of Chemistry, Cornell University (1955).

[12] J. Goubeat, M. RAhtz and H. J. Becher, Z. anorg. allgem. Chem. 275, 11 (1954).

[13] E. O. Camble, Inorganic Syntheses, III, p. 27. McGraw-Hill, New York (1950). 
complex in excess diethyl ether. The solid complex which precipitated was separated from excess solvent, dissolved in cold absolute methanol, and passed through a short column of activated charcoal. Sufficient methanol was then pumped from the filtrate to cause partial precipitation of solid which was separated from the mother liquor and dried in vacuum to remove traces of solvent. Recrystallization from hot alcohol was tried but spectroscopic examination showed decomposition had occurred to give tetrafluoborate ion as one of the products. The same or a similar decomposition was found to occur very slowly when the complex was allowed to stand and consequently spectra were obtained from freshly purified material.

Adducts of TMA with the normal and enriched boron trichloride and tribromide were prepared by direct combination of the reactants in benzene [6, 7]. The products were recrystallized from absolute ethanol in a manner similar to that for the $\mathbf{B F}_{3}$ complex and excess solvent was removed in vacuum. The following melting points were observed: TMA: $\mathrm{BF}_{3}, 144-146^{\circ} \mathrm{C}$, lit. $[2,3], 138-147^{\circ} \mathrm{C}$; $\mathrm{TMA}^{\circ} \mathrm{BCl}_{3}, 241-243^{\circ} \mathrm{C}$, lit. [6]. $242-243^{\circ} \mathrm{C}$; $\mathrm{TMA}: \mathrm{BBr}_{3}, 234-236^{\circ} \mathrm{C}$, lit. [7], 238-240 ${ }^{\circ} \mathrm{C}$. Powder X-ray diffraction data of the boron trifluoride complex agreed with values calculated from the single crystal data [8].

\section{Spectroscopic techniques}

Infrared spectra were recorded using a Perkin-Elmer Model 21 spectrophotometer equipped with $\mathrm{CaF}_{2}, \mathrm{NaCl}$ and $\mathrm{KBr}$ prisms over the range between 400 and $3500 \mathrm{~cm}^{-1}$. Calibration of each spectrum was made with an indene-camphorcyclohexanone standard [14]. The low frequency region was also examined using a Beckman IR-4 equipped with a $\mathrm{CsBr}$ prism through the courtesy of American Cyanamid Company. All samples were prepared in $\mathrm{KBr}$ dises using precautions to eliminate traces of water. Several concentrations were employed for each compound to provide the best rendition of both high and low intensity bands.

Raman spectra of the solid complexes were obtained by pressing the pure crystalline powders into dises 1-3 $\mathrm{mm}$ thick using the same die as for the $\mathrm{KBr}$ pellets. These semi-translucent dises were illuminated on one side by spectrally pure $\mathrm{Hg} 4358$ radiation and the light from the opposite side was collected and passed into the spectrograph. The light source consisted of an AH-4 mercury arc lamp which provided a fairly small are of high intrinsic intensity but without such an extremely high continuous background that it could not be removed by filtering. Both absorption and interference type filters were used for the latter purpose and the spectral purity of the exciting light was judged as good or better than that customarily employed for the Raman effect of liquids using a Toronto are and standard filters. The excess exciting light accompanying the Raman frequencies was reduced significantly before entering the spectrograph by multiple reflections from interference filters $[15,16]$. The thickness of the sample which, in this method, determines the intensity of the Raman scattering [17] was adjusted to an optimum value by trial and

[14] R. N. Jones, N. B. W. Jonathan, M. A. Mackminge and A. Nanfad, Spectrochim, Acta 1\%, $77(1961)$.

[15] J. Brandmuleer, Z. angew. Phys. 5, 95 (1953).

[16] B. Schrader, F. Nerde and G. Kresze, Z. phys. Chem. 12, 132 (1957).

[17] M. C. Tobin, J. Opt. Soc. Am. 49, 850 (1959). 
error. Spectra were recorded photographically using Eastman IIa-O spectrographic plates whose sensitivity had been increased by preheating at $60^{\circ} \mathrm{C}$ for $12-24 \mathrm{hr}$ under anhydrous conditions. Wavelength measurements of the Raman lines were made both on plates and microphotometer tracings. The tabulated data represent mean values obtained from several spectra. In the case of $\mathrm{BCl}_{3}$ and $\mathrm{BBr}_{3}$ complexes, spectra of the loose crystals also were obtained using the instrument at the American Cyanamid Company [17]. These results were particularly valuable in the low frequency region below about $400 \mathrm{~cm}^{-1}$.

\section{Experimental Results}

The experimental frequencies and assignments for the trimethylamine complexes of normal and isotopic varieties of boron trifluoride, boron trichloride and boron tribromide are listed respectively in Tables 1,2 and 3. Infrared spectra of the compounds are reproduced in Figs. 1 and 2 in which the spectra chosen for presentation were selected to show the principal features most clearly. Many more frequencies were observed for all compounds than have previously been reported. The agreement between the infrared and Raman results in the present study is quite satisfactory, the small differences found being no more than might be expected from experimental error.

The infrared spectra of the $\mathrm{BF}_{3}$ and $\mathrm{BCl}_{3}$ complexes (Fig. 1) show good qualitative agreement with the tracings of PETERson and BAUER [11], with due allowance for the difference in resolution. However, marked disagreement was found with the Raman data reported by GoubEaU [12] for the $\mathrm{BCl}_{3}$ complex. In particular, the four strongest bands observed in the present investigation at 383, 535, 826 and $967 \mathrm{~cm}^{-1}$ are all missing in his list of frequencies and it is possible that the substance he examined may not have been sufficiently well characterized. Literature results for the $\mathrm{BBr}_{3}$ complex are rather limited but agreement was noted for the few values available.

\section{Discussion oh Assignmen's}

Complexes of the formula $\left(\mathrm{CH}_{3}\right)_{3} \mathrm{NBX} \mathrm{X}_{3}$ possess 45 fundamental vibrations which, on the basis of $C_{3 v}$ symmetry, comprise $10 A_{1}, 5 A_{2}$ and $15 E$ modes. The $A_{1}$ and $E$ frequencies are allowed in both the Raman and infrared, while the $A_{2}$ fundamentals are inactive in both. The classification, numbering and a simplified description of the various fundamentals are given in Table 4.

From a spectroscopic point of view, the study of Lewis complexes has an advantage in that the formation of the dative bond perturbs but does not radically change the nature of the interacting species. Since the structural study of the $\mathrm{BF}_{3}$ complex [8] has shown that the configuration of the amine half of the compound does not differ markedly from that of the free base, a similarity in the frequencies of the free and combined amine is to be expected. On the other hand, the distortion of the planar structure of the free boron halides plus changes in their force constants caused by rehybridization may. be expected to alter the vibrational frequencies of the $\mathrm{BX}_{3}$ group significantly. In both cases, the magnitude of the frequency shifts can serve as a qualitative indication of the extent of perturbation of the two groups resulting from the formation of the dative bond. 
Table 1. Infrared and Raman frequencies observed for various isotopic varieties of $\left(\mathrm{CH}_{3}\right)_{3} \mathrm{NBF}_{3}$ (in $\mathrm{cm}^{-1}$ )

\begin{tabular}{|c|c|c|c|c|}
\hline \multicolumn{2}{|c|}{ Natural $\left(81 \% \mathrm{~B}^{11}\right)$ Boron } & \multirow[b]{2}{*}{ Assignment } & \multicolumn{2}{|c|}{$92 \% \mathrm{~B}^{10}$ Enriched } \\
\hline $\begin{array}{c}\text { Infrared } \\
\text { (KBr pellet) }\end{array}$ & $\begin{array}{c}\text { Raman } \\
\text { (crystal) }\end{array}$ & & $\begin{array}{c}\text { Infrared } \\
\text { (KBr pellet) }\end{array}$ & $\begin{array}{c}\text { Raman } \\
\text { (crystal) }\end{array}$ \\
\hline $3033 \mathrm{w}$ & $3030 \mathrm{~s}$ & $\nu_{16}$ & $3035 \mathrm{w}$ & $3035 \mathrm{~s}$ \\
\hline $3013 \mathrm{~m}$ & & $\nu_{17}$ & 3016 & \\
\hline $2971 \mathrm{sh}$ & & $v_{19}+v_{20}$ & $2976 \mathrm{vw}$ & \\
\hline $\begin{array}{l}2954 \mathrm{~s} \\
2924 \mathrm{vw}\end{array}$ & $2967 \mathrm{~s}$ & $\nu_{1}$ & $\begin{array}{l}2956 \mathrm{~s} \\
2925 \mathrm{vw}\end{array}$ & $2967 \mathrm{~s}$ \\
\hline $2890 \mathrm{w}$ & $2889 \mathrm{~m}$ & $v_{18}$ & $2890 \mathrm{w}$ & $2885 \mathrm{~m}$ \\
\hline $2868 \mathrm{vw}$ & & & $2868 \mathrm{vw}$ & \\
\hline 2845 vw & & & $2845 \mathrm{vw}$ & \\
\hline $2807 \mathrm{vw}$ & $2802 \mathrm{sh}$ & $v_{2}$ & $2807 \mathrm{vw}$ & $2806 \mathrm{sh}$ \\
\hline \multicolumn{2}{|l|}{$99 \% \mathbf{B}^{11}$ enriched } & \multicolumn{3}{|c|}{$92 \% \mathrm{~B}^{10}$ enriched } \\
\hline $1487 \mathrm{~m}$ & & $v_{19}$ & $1487 \mathrm{~m}$ & \\
\hline $1480 w$ & & $\nu_{20}$ & $1480 \mathrm{w}$ & \\
\hline $1469 \mathrm{w}$ & $1473 \mathrm{vs}$ & $v_{3}$ & $1470 \mathrm{w}$ & $1473 \mathrm{vs}$ \\
\hline $1454 \mathrm{w}$ & & & $1457 \mathrm{w}$ & \\
\hline $1112 \mathrm{vw}$ & $1113 \mathrm{vw}$ & $v_{4}$ & & $1420 \mathrm{vw}$ \\
\hline $1271 \mathrm{vw}$ & & $\nu_{6}+\nu_{26}$ & $1270 \mathrm{sh}$ & \\
\hline $1255 \mathrm{w}$ & $1253 w$ & $v_{22}$ & $1255 \mathrm{w}$ & $125.5 \mathrm{w}$ \\
\hline $1208 \mathrm{w}$ & & & $1211 \mathrm{w}$ & \\
\hline $\begin{array}{l}1175 \mathrm{sh} \\
1142 \mathrm{~s}, \mathrm{br}\end{array}$ & $1178 \mathrm{vvw}$ & $\begin{array}{l}\nu_{6}-\nu_{27} \\
\nu_{25}\end{array}$ & $\begin{array}{l}1180 \mathrm{sh} \\
1165 \mathrm{~s}, \mathrm{br}\end{array}$ & $\begin{array}{l}1178 \mathrm{vvw} \\
1165 \mathrm{vvw}\end{array}$ \\
\hline $1142 \mathrm{~s}$, br & & & $\begin{array}{l}1165 \mathrm{~s}, \mathrm{br} \\
1136 \mathrm{vvw}\end{array}$ & \\
\hline & & & $1122 \mathrm{vw}$ & \\
\hline $1104 \mathrm{~m}$ & $1105 \mathrm{w}$ & $\nu_{23}$ & $1109 \mathrm{w}$ & $1114 \mathrm{w}$ \\
\hline $1017 \mathrm{vw}$ & & $\nu_{7}+\nu_{9}$ & $1014 \mathrm{vw}$ & \\
\hline $991 \mathrm{w}$ & $988 \mathrm{~s}$ & $v_{34}$ & $987 \mathrm{w}$ & $991 \mathrm{~s}$ \\
\hline $932 \mathrm{~s}$ & $935 \mathrm{w}, \mathrm{sh}$ & $\nu_{8}$ & $952 \mathrm{~s}$ & \\
\hline $841 \mathrm{~s}$ & $842 \mathrm{~s}$ & $v_{6}$ & $838 \mathrm{~m}$ & $847 \mathrm{~s}$ \\
\hline $692 \mathrm{~m}$ & $694 \mathrm{~s}$ & $\nu_{7}$ & $696 \mathrm{~m}$ & $700 \mathrm{~s}$ \\
\hline $640 \mathrm{vW}$ & & $2 \nu_{9}$ & 643 vvw & $640 \mathrm{w}$ \\
\hline $547 \mathrm{w}$ & $545 \mathrm{vw}$ & $\nu_{10}$ & $\begin{array}{l}550 \mathrm{w} \\
536 \mathrm{vw}\end{array}$ & $545 \mathrm{vw}$ \\
\hline $519 \mathrm{vw}, \mathrm{sh}$ & $5 \mathrm{l} 3 \mathrm{vw}$ & $2 v_{30} ?$ & $525 \mathrm{w}$ & $518 \mathrm{vw}$ \\
\hline $464 \mathrm{vvw} ?$ & & & 465 vvw? & \\
\hline $430 w$ & $\begin{array}{l}428 \text { vVw } \\
372 \times w\end{array}$ & $\begin{array}{c}v_{26} \\
?\end{array}$ & $431 \mathrm{w}$ & $429 \mathrm{vw}$ \\
\hline $340 \mathrm{w}$ & & $\nu_{27}$ & $340 \mathrm{w}$ & \\
\hline $320 \mathrm{w}$ & & $\nu_{\theta}$ & $323 \mathrm{vw}$ & \\
\hline
\end{tabular}

\section{Methyl group frequencies}

The bands associated with the stretching and deformation motions of the methyl groups in the complexes are well isolated from the rest of the frequencies and a detailed discussion of their assignments does not appear necessary. As commonly happens, the overtones of the methyl deformation frequencies resonate with the $\mathrm{C}-\mathrm{H}$ stretching fundamentals to complicate the pattern of bands in the $2900 \mathrm{~cm}^{-1}$ region. Complexing might be expected to have relatively little effect on these frequencies but it is interesting to note that the formation of the dative bond apparently changes the degree of coupling between the methyl deformation modes so that the accidental degeneracy present in the free amine is reduced in the complex. This effect is most pronounced in the $\mathrm{BCl}_{3}$ case. The methyl group rocking modes are relatively well isolated between 1100 and $1270 \mathrm{~cm}^{-1}$ except in the $\mathrm{BF}_{3}$ complex whose spectrum in this region contains a $\mathrm{B}-\mathrm{F}$ stretching mode plus overtones. The B-F fundamental is identifiable by its intensity, sensitivity to isotopic substitution 
Table 2. Infrared and Raman frequencies observed for normal and $\mathrm{B}^{10}$ enriched varieties of $\left(\mathrm{CH}_{3}\right)_{3} \mathrm{NBCl}_{3}$ (in $\mathrm{cm}^{-1}$ )

\begin{tabular}{|c|c|c|c|c|}
\hline \multicolumn{2}{|c|}{ Natural boron } & \multirow[b]{2}{*}{ Assignment } & \multicolumn{2}{|c|}{$92 \% \mathrm{~B}^{10}$ enriched } \\
\hline $\begin{array}{c}\text { Infrared } \\
\text { (KBr pellet) }\end{array}$ & $\begin{array}{l}\text { Raman } \\
\text { (crystal) }\end{array}$ & & $\begin{array}{c}\text { Infrared } \\
\text { (KBr pellet) }\end{array}$ & $\begin{array}{c}\text { Raman } \\
\text { (crystal) }\end{array}$ \\
\hline 3479 vvw & & $v_{16}+v_{26}$ & $3470 \mathrm{vvw}$ & \\
\hline $3417 \mathrm{vvw}$ & & $v_{8}+v_{18}$ & $3425 \mathrm{vvw}$ & \\
\hline $3360 \mathrm{vvw}$ & & $v_{9}+v_{17}$ & & \\
\hline 3305 vvw & & $v_{1}+v_{9}$ & 3305 vvw & \\
\hline $3233 \mathrm{vvw}$ & & $v_{1}+v_{27}$ & $3233 \mathrm{vvw}$ & \\
\hline $3075 \mathrm{vw}$ & & $v_{18}+v_{29}, v_{2}+v_{27}$ & 3072 vw & \\
\hline $3044 \mathrm{w}$ & $3035 \mathrm{~s}$ & $v_{16}$ & $3043 \mathrm{w}$ & $3035 \mathrm{~s}$ \\
\hline $3026 \mathrm{w}$ & $3020 \mathrm{sh}$ & $y_{17}$ & $3025 \mathrm{w}$ & $3023 \mathrm{sh}$ \\
\hline 2972 sh & & $2 v_{19}$ & & \\
\hline $2964 \mathrm{~m}$ & $2966 \mathrm{~s}$ & $\nu_{1}$ & $2962 \mathrm{~m}$ & $2965 \mathrm{~s}$ \\
\hline $2922 \mathrm{w}$ & $2922 \mathrm{vw}$ & $v_{3}+v_{20}$ & $2922 \mathrm{w}$ & $2922 \mathrm{vw}$ \\
\hline $2889 \mathrm{w}$ & $2887 \mathrm{w}$ & $v_{18}$ & $2889 \mathrm{w}$ & $2888 \mathrm{w}$ \\
\hline $2843 w$ & & $2 v_{4}^{*}$ & $2842 \mathrm{w}$ & \\
\hline $2804 \mathrm{vw}$ & $2802 \mathrm{w}$ & $v_{2}$ & $2804 \mathrm{vw}$ & $2806 \mathrm{w}$ \\
\hline $2748 \mathrm{vw}$ & & $v_{10}+v_{22}$ & $2745 \mathrm{vw}$ & $2740 \mathrm{vw}$ \\
\hline $2713 \mathrm{vvw}$ & & $v_{3}+v_{22}, v_{5} \div v_{19}$ & $2715 \mathrm{vvw}$ & \\
\hline $2694 \mathrm{vvw}$ & & $\nu_{5}+v_{20}$ & $2694 \mathrm{vvW}$ & \\
\hline $2677 \mathrm{vw}$ & & $v_{21}+v_{22}$ & $2677 \mathrm{vw}$ & \\
\hline $2555 \mathrm{vW}$ & & $\nu_{3}-v_{23}$ & $2557 \mathrm{vw}$ & \\
\hline $2521 \mathrm{vw}$ & & $2 v_{22}, v_{4}+v_{23}$ & $2521 \mathrm{w}$ & \\
\hline $2461 \mathrm{vw}$ & & $2 v_{5}$ & $2461 \mathrm{vw}$ & \\
\hline $2426 \mathrm{vvw}$ & & $v_{1}-v_{8}$ & $2427 \mathrm{vvw}$ & \\
\hline $2367 w$ & & $v_{4}+v_{24}$ & $2371 \mathrm{w}$ & \\
\hline $2235 \mathrm{w}$ & & $v_{4}+v_{6}$ & $2238 \mathrm{w}$ & \\
\hline $2231 \mathrm{sh}$ & & $2 v_{23}$ & $2229 \mathrm{sh}$ & \\
\hline $2196 \mathrm{vvw}$ & & $\nu_{5}+\nu_{24}$ & $2193 \mathrm{vvw}$ & \\
\hline $2155 \mathrm{vvw}$ & & $\nu_{4}+\nu_{7}$ & $2181 \mathrm{vvw}$ & \\
\hline $2055 \mathrm{vvW}$ & * & $v_{5}+v_{6}$ & & \\
\hline $2002 \mathrm{vvw}$ & " & $\begin{array}{l}v_{7}\left(\mathrm{~B}^{10}\right)+v_{22} \\
v_{8}+v_{90}\end{array}$ & $2028 \mathrm{vVw}$ & \\
\hline $1980 \mathrm{vvw}$ & & $\begin{array}{l}v_{8}+v_{20} \\
v_{3}+v_{8}\end{array}$ & $2000 \mathrm{vvw}$ & \\
\hline 1942 vvw & & $v_{6}+v_{23}$ & $1945 \mathrm{vvw}$ & \\
\hline $1923 \mathrm{vw}$ & & $2 v_{24}, v_{19} \div v_{26}$ & $1926 \mathrm{vw}$ & \\
\hline $1890 \mathrm{vvw}$ & & $\nu_{3}+v_{26}$ & $1892 \mathrm{vvw}$ & \\
\hline $1792 \mathrm{vw}$ & & $\nu_{6}+v_{24}$ & $1795 \mathrm{vw}$ & \\
\hline $1734 \mathrm{vw}$ & & $\nu_{20}+\nu_{28}$ & $1734 \mathrm{vw}$ & \\
\hline $1710 \mathrm{vw}$ & & $\nu_{20}+\nu_{29}$ & $1712 \mathrm{vw}$ & \\
\hline $1680 \mathrm{vvw}$ & & $v_{5}+v_{26}$ & $1680 \mathrm{vvw}$ & \\
\hline $1650 \mathrm{vvw}$ & & $v_{8}+v_{23}$ & $1625 \mathrm{vvw}$ & \\
\hline $1571 \mathrm{vw}$ & & $\nu_{6}+\nu_{7}$ & $1598 \mathrm{vw}$ & \\
\hline $1553 \mathrm{vvw}$ & & $v_{23}+v_{26}$ & $1553 \mathrm{vvw}$ & \\
\hline $1506 \mathrm{sh}$ & & $\nu_{8}+\nu_{24}$ & $1506 \mathrm{sh}$ & \\
\hline $1485 \mathrm{~s}$ & $1481 \mathrm{sh} ?$ & $\nu_{19}$ & $1486 \mathrm{~s}$ & $1481 \mathrm{sh} ?$ \\
\hline $1465 \mathrm{~s}$ & $1459 \mathrm{sh} ?$ & $v_{20}$ & $1463 \mathrm{~s}$ & \\
\hline $1451 \mathrm{~m}$ & $1447 \mathrm{~s}$ & $v_{3}$ & $1451 \mathrm{~m}$ & $1449 \mathrm{~s}$ \\
\hline $1412 \mathrm{~m}$ & & $v_{21}$ & $1412 \mathrm{~m}$ & \\
\hline $1408 \mathrm{~m}$ & $1409 \mathrm{vw}$ & $v_{4}$ & $1408 \mathrm{~m}$ & $1410 \mathrm{vw}$ \\
\hline $1344 \mathrm{vw}$ & & $v_{10}+v_{24}$ & $1344 \mathrm{vw}$ & \\
\hline
\end{tabular}


Table 2 (contd.)

\begin{tabular}{|c|c|c|c|c|}
\hline \multicolumn{2}{|c|}{ Natural boron } & \multirow[b]{2}{*}{ Assignment } & \multicolumn{2}{|c|}{$92 \% \mathrm{~B}^{10}$ enriched } \\
\hline $\begin{array}{c}\text { Infrared } \\
\text { (KBr pellet) }\end{array}$ & $\begin{array}{l}\text { Raman } \\
\text { (crystal) }\end{array}$ & & $\begin{array}{c}\text { Infrared } \\
(\mathrm{KBr} \text { pellet })\end{array}$ & $\begin{array}{c}\text { Raman } \\
\text { (crystal) }\end{array}$ \\
\hline $1263 \mathrm{~m}$ & $1263 \mathrm{vW}$ & $v_{22}$ & $1264 \mathrm{~m}$ & \\
\hline $1232 \mathrm{~s}$ & $1231 \mathrm{w}$ & $v_{5}$ & $1232 \mathrm{~s}$ & $1232 \mathrm{w}$ \\
\hline $1116 \mathrm{~s}$ & $1115 \mathrm{w}$ & $v_{23}$ & $1116 \mathrm{~s}$ & $1116 \mathrm{w}$ \\
\hline $976 \mathrm{sh}$ & & $v_{8}+v_{26}$ & $976 \mathrm{sh}$ & \\
\hline 965 vs & $967 \mathrm{~s}$ & $v_{24}$ & $965 \mathrm{vs}$ & $968 \mathrm{~s}$ \\
\hline 837 sh? & & & 840 sh? & \\
\hline $833 \mathrm{vs}$ & $826 \mathrm{~s}$ & $v_{6}$ & $835 \mathrm{vs}$ & $828 \mathrm{~s}$ \\
\hline $786 \mathrm{~m}$ & & $v_{25}\left(\mathrm{~B}^{10}\right)$ & 783 vs & \\
\hline & $750-790$ & $v_{7}\left(\mathrm{~B}^{10}\right)$ & $770 \mathrm{~m}$ & $773 \mathrm{~m}$ \\
\hline $765 \mathrm{sh} ?$ & & $2 v_{10}$ & $761 \mathrm{w}$ & \\
\hline $755 \mathrm{vs}$ & & $v_{25}\left(\mathrm{~B}^{11}\right)$ & $757 \mathrm{vw} ?$ & \\
\hline $746 \mathrm{~m}$ & $744 \mathrm{~ms}$ & $\nu_{7}\left(\mathrm{~B}^{11}\right)$ & $748 w$ & \\
\hline $721 \mathrm{w}$ & & $v_{26}+v_{27}, v_{9} \div v_{10}$ & $721 \mathrm{w}$ & \\
\hline $650 \mathrm{vw}$ & & $v_{10}+v_{27}$ & $648 \mathrm{vw}$ & \\
\hline $538 \mathrm{vw}$ & $535 \mathrm{~s}$ & $v_{8}$ & $540 \mathrm{vw}$ & 536 s \\
\hline $443 \mathrm{~m}$ & $445 \mathrm{w}$ & $\nu_{26}$ & $443 \mathrm{~m}$ & $445 \mathrm{w}$ \\
\hline $381 \mathrm{w}$ & $383 \mathrm{~s}$ & $v_{10}$ & $381 \mathrm{w}$ & $383 \mathrm{~s}$ \\
\hline $337 \mathrm{~m}$ & $332 \mathrm{w}$ & $v_{9}$ & $337 \mathrm{~m}$ & $333 \mathrm{sh}$ \\
\hline & $271 w$ & $y_{27}$ & & $269 \mathrm{w}$ \\
\hline & $255 \mathrm{w}$ & $\nu_{28}$ or $\nu_{29}$ & & $256 \mathrm{w}$ \\
\hline & $183 \mathrm{w}$ & $v_{29}$ or $v_{30}$ & & $183 \mathrm{w}$ \\
\hline
\end{tabular}

$\mathrm{s}=$ strong, $\mathrm{m}=$ medium, $\mathrm{w}=$ weak, $\mathrm{sh}=$ shoulder, ete; $*$ perturbed by Fermi resonance

and by comparison with the spectra of the other complexes. Assignments of the methyl rocking modes to some extent are arbitrary but have been made on the basis of the assignments for the free amine. Overlapping occurs in the case of the $\mathrm{BF}_{3}$ complex, and the $\mathrm{B}^{10}$ and $\mathrm{B}^{11}$ spectra of this compound show characteristic differences in the $1100 \mathrm{~cm}^{-1}$ region.

\section{Amine frequencies}

Vibrational frequencies and assignments for free trimethyl amine are available in the literature $[18,19]$ and appear reasonably well established. As expected, the corresponding frequencies of the complexes were easy to identify since they were not markedly shifted in position. The symmetric $\mathrm{C}-\mathrm{N}$ stretching mode at $826 \mathrm{~cm}^{-1}$ in the free amine shifts to $842 \mathrm{~cm}^{-1}$ in the $\mathrm{BF}_{3}$ complex, but is observed at about $830 \mathrm{~cm}^{-1}$, essentially unaffected, in the other two compounds. KaTRITSkY [10] assigned this hand to the asymmetric $\mathrm{B}-\mathrm{Cl}$ stretch in the chloride complex but, in view of the evidence from the other two complexes plus the lack of a significant isotope effect, this does not appear to be correct. The asymmetric $\mathrm{C}-\mathrm{N}$ stretching fundamental, $v_{24}$, is more sensitive to the attaching group, the band at $1043 \mathrm{~cm}^{-1}$ in the 
Table 3. Infrared and Raman frequencies observed for normal and $\mathrm{B}^{\mathbf{1 0}}$ enriched varieties of $\left(\mathrm{CH}_{3}\right)_{3} \mathrm{NBBr}_{3}$ (in $\left.\mathrm{cm}^{-1}\right)$

\begin{tabular}{|c|c|c|c|c|}
\hline \multicolumn{2}{|c|}{ Natural boron } & \multirow[b]{2}{*}{ Assignment } & \multicolumn{2}{|c|}{$92 \% \mathrm{~B}^{10}$ enriched } \\
\hline $\begin{array}{c}\text { Infrared } \\
\text { (KBr pellet) }\end{array}$ & $\begin{array}{l}\text { Raman } \\
\text { (erystal) }\end{array}$ & & $\begin{array}{c}\text { Infrared } \\
\text { (KBr pellet) }\end{array}$ & $\begin{array}{c}\text { Raman } \\
\text { (orystal) }\end{array}$ \\
\hline $3061 \mathrm{vvw}$ & & $\nu_{18}+\nu_{29}$ & 3063 vvw & \\
\hline $3036 w$ & $3030 \mathrm{~m}$ & $v_{16}$ & $3036 \mathrm{w}$ & $3025 \mathrm{~m}$ \\
\hline $3021 \mathrm{vw}$ & & $v_{17}$ & $\begin{array}{l}3023 \mathrm{vw} \\
3014 \mathrm{vw}\end{array}$ & \\
\hline $2956 \mathrm{~s}$ & $2960 \mathrm{~s}$ & $\nu_{1}$ & $2954 \mathrm{~m}$ & $2957 \mathrm{~s}$ \\
\hline $2948 \mathrm{vw}$ & & $v_{19}+v_{20}$ & & \\
\hline $2919 \mathrm{vvw}$ & $2912 \mathrm{vw}$ & $2 v_{20}$ & $2917 \mathrm{vw}$ & $2915 \mathrm{vVw}$ \\
\hline $\begin{array}{l}2882 \mathrm{w} \\
2851 \mathrm{vw}\end{array}$ & $2883 \mathrm{w}$ & $\begin{array}{l}v_{18} \\
v_{3}+v_{4}\end{array}$ & $2882 \mathrm{w}$ & $2880 \mathrm{w}$ \\
\hline $2798 \mathrm{vw}$ & $2797 ?$ & $v_{2}$ & $2797 \mathrm{vw}$ & \\
\hline $2729 \mathrm{vvw}$ & & $v_{20}+v_{22}$ & $2731 \mathrm{vvw}$ & \\
\hline $2698 w$ & & $v_{3}+v_{22}$ & & \\
\hline $2664 \mathrm{vw}$ & & $v_{4}+v_{22}$ & $2664 \mathrm{vw}$ & \\
\hline $2549 \mathrm{vw}$ & & $v_{3}+\nu_{23}$ & $2550 \mathrm{vW}$ & \\
\hline $2512 \mathrm{vw}$ & & $2 v_{22}$ & $2512 \mathrm{vw}$ & \\
\hline & & & 2496 vvw & \\
\hline $2465 \mathrm{vvw}$ & & & $2465 \mathrm{vvw}$ & \\
\hline $2445 \mathrm{vvw}$ & & $v_{19} \mid v_{24}$ & $2445 \mathrm{vvw}$ & \\
\hline 2413 vvw & & $v_{20}+v_{24}$ & $2413 \mathrm{vvw}$ & \\
\hline $2397 \mathrm{vVw}$ & & $v_{3}+\nu_{24}$ & $2397 \mathrm{vvw}$ & \\
\hline $2347 \mathrm{vw}$ & & & $2351 \mathrm{vw}$ & \\
\hline & & $v_{4}+v_{6}$ & $2234 \mathrm{sh}$ & \\
\hline $2223 \mathrm{vw}$ & & $2 v_{23}$ & $2223 \mathrm{vw}$ & \\
\hline 2130 vvw & & $v_{3}+v_{25}$ & $2154 \mathrm{vvw}$ & \\
\hline & & $v_{5}+v_{19}$ & 2005 vvw & \\
\hline $1982 \mathrm{vvw}$ & & $v_{8}+\nu_{20}$ & & \\
\hline $1953 \mathrm{vvw}$ & & $v_{7}+v_{22}$ & 1972 vvw & \\
\hline $1908 \mathrm{vvw}$ & & $2 v_{24}$ & $1909 \mathrm{vvw}$ & \\
\hline & & $v_{3}+v_{26}$ & $1879 \mathrm{vvw}$ & \\
\hline $1762 \mathrm{vvw}$ & & $v_{3}+v_{9}$ & $1770 \mathrm{vvw}$ & \\
\hline & & $\nu_{5}+v_{8}$ & $1745 \mathrm{vvw}$ & \\
\hline & & $v_{3}+v_{10} ?$ & $1706 \mathrm{vw}$ & \\
\hline $1588 \mathrm{vvw}$ & & $v_{9}+\nu_{22}$ & $1574 \mathrm{vw}$ & \\
\hline & & & $1559 \mathrm{vvw}$ & \\
\hline $1546 \mathrm{vw}$ & & $v_{23}+v_{26}$ & & \\
\hline $1540 \mathrm{sh}$ & & $\nu_{5}+v_{9}$ & $1539 \mathrm{vvw}$ & \\
\hline $1522 \mathrm{vvw}$ & & $v_{10}+v_{22}$ & $1520 \mathrm{vvw}$ & \\
\hline $1484 \mathrm{~s}$ & $1484 \mathrm{rw}, \mathrm{sh}$ & $\nu_{19}$ & $\begin{array}{l}1483 \mathrm{~s} \\
1476 \mathrm{sh}\end{array}$ & $1480 \mathrm{vw}, \mathrm{sh}$ \\
\hline $1461 \mathrm{~s}$ & $1460 \mathrm{sh}$ & $v_{20}$ & $1461 \mathrm{~s}$ & \\
\hline $1448 \mathrm{~m}$ & $1448 \mathrm{~s}$ & $\nu_{3}$ & $1448 \mathrm{~m}$ & $1448 \mathrm{~s}$ \\
\hline $1410 \mathrm{~s}$ & 1408 vvw & $v_{4}, v_{21}$ & $1409 \mathrm{~s}$ & $1408 \mathrm{vvw}$ \\
\hline $1345 \mathrm{vw}$ & $1345 \vee v w$ & $\nu_{6}+\nu_{8}$ & $1346 \mathrm{vw}$ & \\
\hline $1260 \mathrm{w}$ & & $y_{22}$ & $1259 \mathrm{w}$ & \\
\hline $1226 \mathrm{~s}$ & $1227 \mathrm{~m}$ & $\nu_{5}$ & $1225 \mathrm{~s}$ & $1227 \mathrm{~m}$ \\
\hline $1165 \mathrm{vw}$ & & $y_{7}+v_{26}$ & & \\
\hline
\end{tabular}


Table 3 (contd.)

\begin{tabular}{|c|c|c|c|c|}
\hline \multicolumn{2}{|c|}{ Natural Boron } & \multirow[b]{2}{*}{ Assignment } & \multicolumn{2}{|c|}{$92 \% \mathrm{~B}^{10}$ enriched } \\
\hline $\begin{array}{c}\text { Infrared } \\
\text { (KBr pellet) }\end{array}$ & $\begin{array}{c}\text { Raman } \\
\text { (crystal) }\end{array}$ & & $\begin{array}{c}\text { Infrared } \\
\text { (KBr pellet) }\end{array}$ & $\begin{array}{c}\text { Raman } \\
\text { (crystal) }\end{array}$ \\
\hline & & $v_{25}+v_{26}$ & $1131 \mathrm{vw}$ & $1140 \mathrm{vw}$ \\
\hline $1113 \mathrm{~s}$ & $1115 \mathrm{w}$ & $v_{23}$ & $1113 \mathrm{~s}$ & $1115 \mathrm{w}$ \\
\hline $1021 \mathrm{vw}$ & & $v_{6}+v_{27}$ & $1020 \mathrm{vw}$ & \\
\hline 957 vs & $959 \mathrm{~s}$ & $v_{24}$ & $955 \mathrm{vs}$ & $960 \mathrm{~s}$ \\
\hline \multirow[t]{2}{*}{$827 \mathrm{vs}$} & $826 \mathrm{~s}$ & $v_{6}$ & $830 \mathrm{vs}$ & $826 \mathrm{~s}$ \\
\hline & & & $772 \mathrm{w}$ & \\
\hline $752 \mathrm{~m}$ & & $\nu_{9}+v_{\mathbf{2 6}}$ & $751 \mathrm{~m}$ & \\
\hline $732 \mathrm{~s}$ & & $v_{8}+v_{27}$ & & \\
\hline \multirow[t]{3}{*}{$707 \mathrm{~s}$} & & $v_{25}\left(\mathrm{~B}^{10}\right)$ & $706 \mathrm{vs}$ & $700 \mathrm{~m}$ \\
\hline & $689 \mathrm{~m}$ & $\nu_{7}$ & & $720 \mathrm{~m}$ \\
\hline & & $v_{25}\left(\mathrm{~B}^{11}\right), v_{8}+v_{29}$ & 678 & \\
\hline $677 \mathrm{vs}$ & $673 \mathrm{~m}$ & $v_{25}\left(\mathrm{~B}^{11}\right)$ & & \\
\hline $518 \mathrm{w}$ & $516 \mathrm{~m}$ & $\nu_{8}$ & $520 \mathrm{w}$ & $518 \mathrm{~m}$ \\
\hline $434 \mathrm{~m}$ & $434 \mathrm{~m}$ & $v_{26}$ & $434 \mathrm{~m}$ & $435 \mathrm{~m}$ \\
\hline \multirow[t]{4}{*}{$316 \mathrm{~m}$} & $318 w$ & $v_{9}$ & $316 \mathrm{~m}$ & $318 w$ \\
\hline & $275 \mathrm{~s}$ & $v_{10}$ & & $275 \mathrm{~s}$ \\
\hline & $194 \mathrm{w}$ & $v_{27}$ & & $195 \mathrm{w}$ \\
\hline & $184 \mathrm{~m}$ & $v_{29}$ & & $182 \mathrm{~m}$ \\
\hline
\end{tabular}

free base shifting successively to 989,965 and $958 \mathrm{~cm}^{-1}$ in the fluoride, chloride and bromide.

The asymmetric $\mathrm{NC}_{3}$ deformation mode shifts slightly to higher frequencies from $425 \mathrm{~cm}^{-1}$ in the free base to 430,443 and $434 \mathrm{~cm}^{-1}$ in the respective complexes, while the symmetric mode decreases in magnitude from $365 \mathrm{~cm}^{-1}$ to 320,337 and $316 \mathrm{~cm}^{-1}$. The isotopic sensitivity of all of these bands was small. It is of interest to note that the symmetric $\mathrm{NH}_{3}$ deformation mode in the spectra of $\mathrm{NH}_{3}$ complexes is significantly higher in frequency than it is in free $\mathrm{NH}_{3}[20,21]$. This is contrary to the relative positions assigned here for the symmetric $\mathrm{NC}_{3}$ deformation but attempts at assigning a frequency to the $\mathrm{NC}_{3}$ mode higher than that observed in the free base clearly led to less satisfactory results.

\section{$\mathrm{BF}_{3}$ group frequencies}

Assignment of frequencies primarily involving the complexed $\mathrm{BF}_{3}$ group can be made by reference to the spcctra of frcc $\mathrm{BF}_{3}$ and of the $\mathrm{BF}_{4}$ - ion. The latter represents the limiting case in which full conversion of the boron to an $s p^{3}$ hybridization and a tetrahedral structure has been achieved. Frequency data for all the boron halides have been summarized by Wentink and TiensuU [22] while the spectra of the $\mathrm{BF}_{4}^{-}$ion have been discussed by Goubeau and BuEs [23], and by

[20] S. I. Mizushima and I. Nakagawa, J. Chem. Phys. 23, 1367 (1955).

[21] R. L. Amster, Dissertation, The University of Michigan (1961).

[22] T. Wentink and V.H. Tiensuu, J. Chem. Phys. 28, 826 (1958).

[23] J. Goubeau and W. Bues, Z. anorg. allgem. Chem. 268, 221 (1952). 


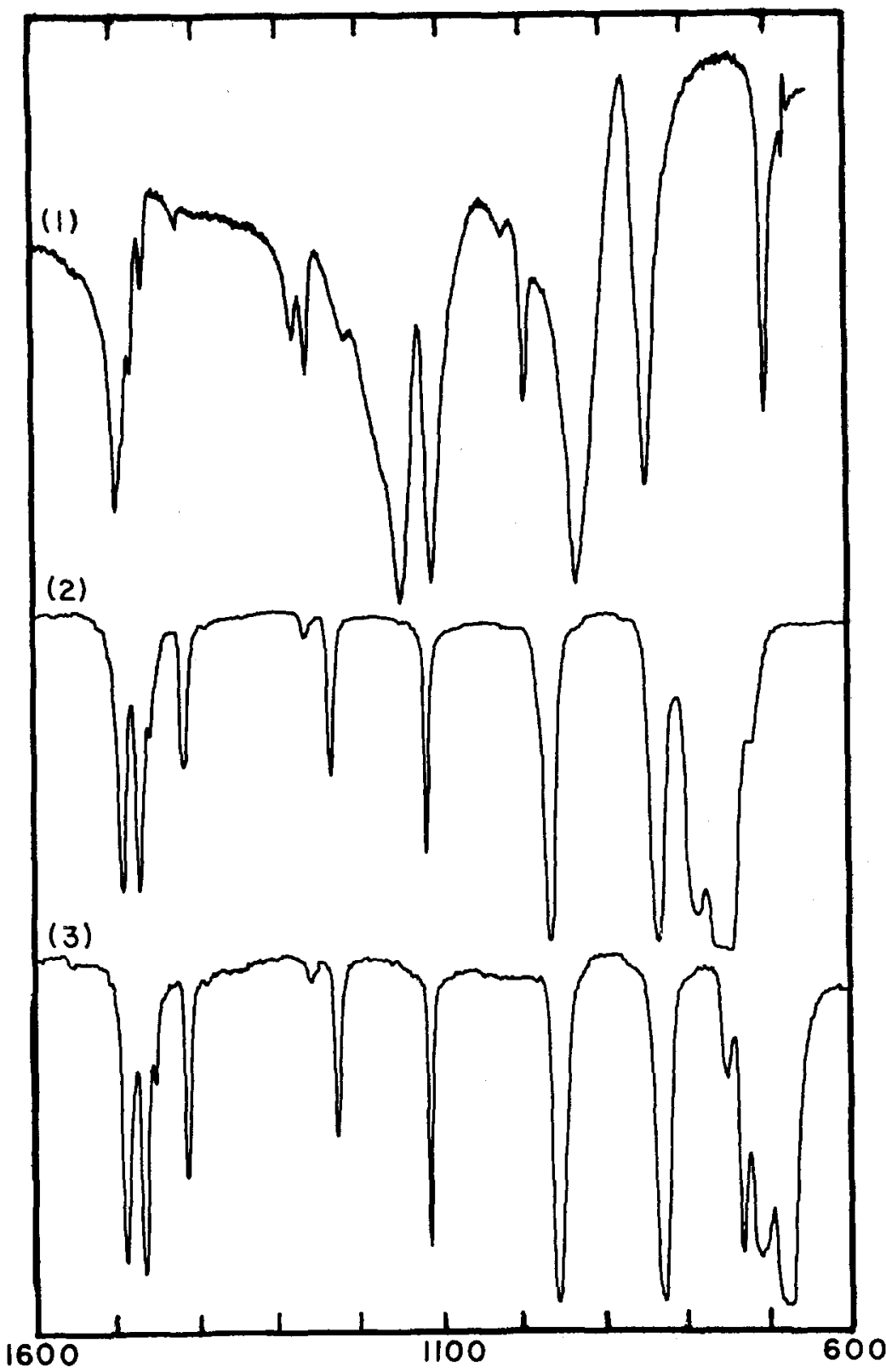

Fig. 1. Infrared spectra of trimethylamine complexes of boron halides in the $\mathrm{NaCl}$ region. (1) $\mathrm{BF}_{3}\left(99 \% \mathrm{~B}^{11}\right),(2) \mathrm{BCl}_{3}\left(81 \% \mathrm{~B}^{11}\right)$, (3) $\mathrm{BBr}_{3}\left(81 \% \mathrm{~B}^{11}\right)$. 


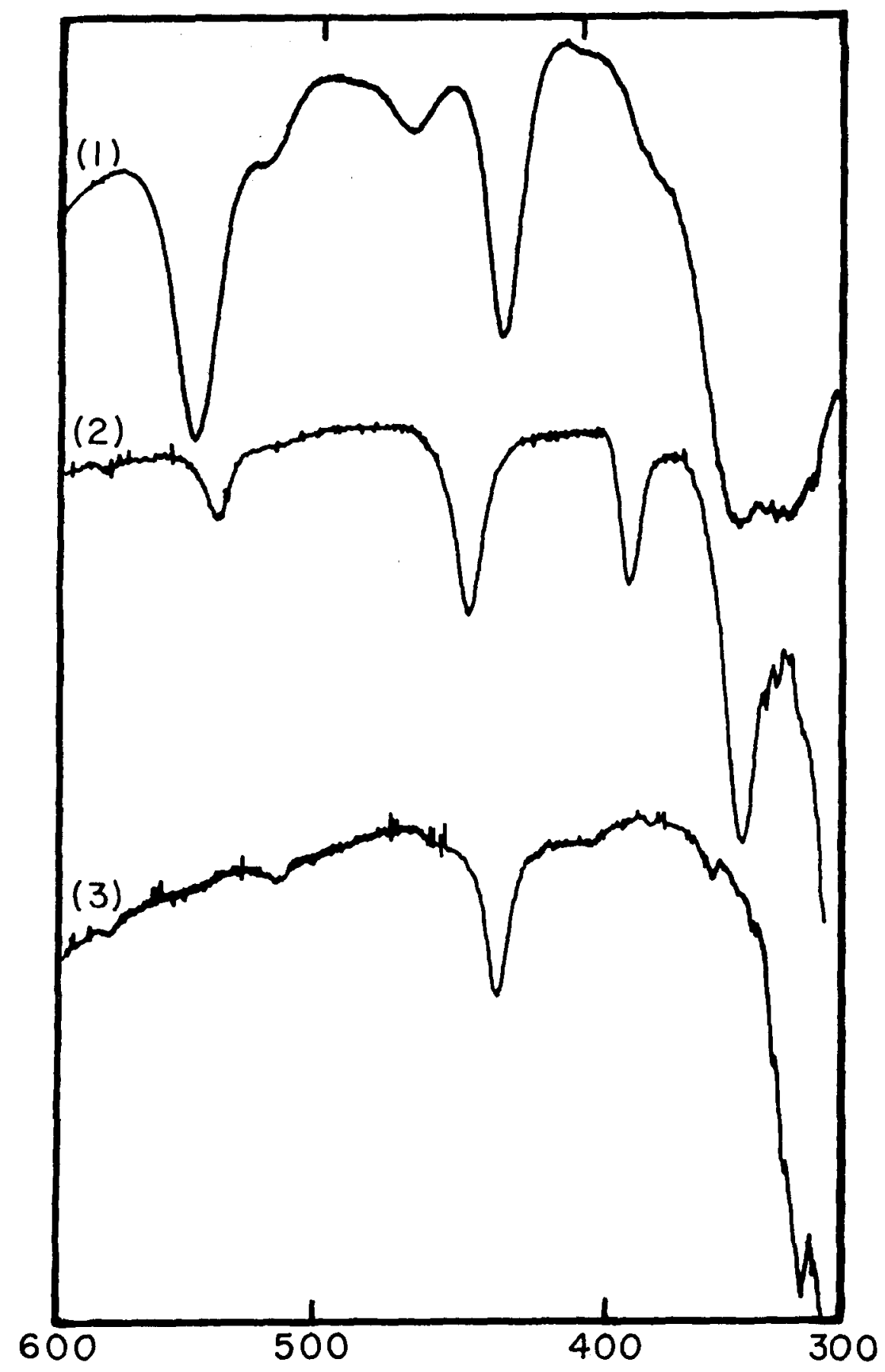

Fig. 2. Infrared spectra of trimethylamine complexes of boron halides in the $\mathrm{KBr}$ region. (1) $\mathrm{BF}_{3}\left(99 \% \mathrm{~B}^{11}\right)$, (2) $\mathrm{BCl}_{3}\left(81 \% \mathrm{~B}^{11}\right)$, (3) $\mathrm{BBr}_{3}\left(81 \% \mathrm{~B}^{11}\right)$. 
Table 4. A description of vibrational modes for $\left(\mathrm{CH}_{3}\right)_{3} \mathrm{~N}: \mathrm{BX}$

\begin{tabular}{|c|c|c|}
\hline Number & Symmetry & Description \\
\hline 1 & $a_{1}$ & $\nu^{s} \mathrm{CH}$-out-of-phase $\mathrm{C}-\mathrm{H}$ stretch \\
\hline 2 & $a_{1}$ & $\nu^{s} \mathrm{CH}$ - in-phase $\mathrm{C}-\mathrm{H}$ stretch \\
\hline 3 & $a_{1}$ & $\delta^{8} \mathrm{CH}_{3}$ - out-of-phase $\mathrm{CH}_{3}$ deformation \\
\hline 4 & $a_{1}$ & $\delta^{s} \mathrm{CH}_{3}{ }_{3}$-in-phase $\mathrm{CH}_{3}$ deformation \\
\hline 5 & $a_{1}$ & $\rho^{s} \mathrm{CH}_{3}-\mathrm{CH}_{3}$ rock \\
\hline 6 & $a_{1}$ & $\nu^{s} \mathrm{CN}-\mathrm{C}-\mathrm{N}$ streteh \\
\hline 7 & $a_{1}$ & $\nu^{s} \mathrm{BN}-\mathrm{B}-\mathrm{N}$ stretch \\
\hline 8 & $a_{1}$ & $v^{3} \mathrm{BX}-\mathrm{B}-\mathrm{X}$ stretch \\
\hline 9 & $a_{1}$ & $\delta^{s} \mathrm{C}_{3} \mathrm{~N}-\overline{\mathrm{C}}_{3} \mathrm{~N}$ deformation \\
\hline 10 & $a_{1}$ & $\delta^{s} \mathbf{B X}{ }_{3}-B_{3}$ deformation \\
\hline 11 & $a_{2}$ & $\nu^{a} \mathrm{CH}$-out-of-phase $\mathrm{C}-\mathrm{H}$ stretch \\
\hline 12 & $a_{2}$ & $\delta^{a} \mathrm{CH}_{3}$-out-of-phase $\mathrm{CH}_{3}$ deformation \\
\hline 13 & $a_{2}$ & $\rho \mathrm{CH}_{3}-\mathrm{CH}_{3}$ rock \\
\hline 14 & $a_{2}$ & ${ }^{2} \mathrm{CH}_{3}-\mathrm{CH}_{3}$ torsion \\
\hline 15 & $a_{2}$ & $\tau \mathrm{BN}$ - torsion about molecular axis \\
\hline 16 & $e$ & $\boldsymbol{v}^{a} \mathrm{CH}-$ out-of-phase $\mathrm{C}-\mathrm{H}$ stretch \\
\hline 17 & $e$ & $v^{\alpha} \mathrm{CH}$-uut-of-phase $\mathrm{C}-\mathrm{H}$ strelch \\
\hline 18 & $e$ & $v^{a} \mathrm{CH}-$ in-phase $\mathrm{C}-\mathrm{H}$ stretch \\
\hline 19 & $e$ & $\delta^{a} \mathrm{CH}_{3}$ out-of-phase $\mathrm{CH}_{3}$ deformation \\
\hline 20 & $e$ & $\delta^{a} \mathrm{CH}_{3}$-out-of-phase $\mathrm{CH}_{3}$ deformation \\
\hline 21 & $e$ & $\delta^{a} \mathrm{CH}_{3}$-in-phase $\mathrm{CH}_{3}$ deformation \\
\hline 22 & $e$ & $\rho \mathrm{CH}_{3}-\mathrm{CH}_{3}$ rock \\
\hline 23 & $e$ & $\omega \mathrm{CH}_{3}^{\circ}-\mathrm{CH}_{3}^{\circ}$ wag \\
\hline 24 & $e$ & $v^{n} \mathrm{CN}-\mathrm{C}-\mathrm{N}$ streteh \\
\hline 25 & $e$ & $\nu^{a} \mathbf{B X}-v^{a} \mathbf{B X}$ stretch \\
\hline 26 & $e$ & $\delta^{a} \mathrm{C}_{3} \mathrm{~N}-\mathrm{C}_{3} \mathrm{~N}$ deformation \\
\hline 27 & $e$ & $\delta^{a} \mathrm{BX}_{3}-\mathrm{BX}_{3}$ deformation \\
\hline 28 & $\boldsymbol{e}$ & $\tau \mathrm{CH}_{3}-\mathrm{CH}_{3}$ torsional motion \\
\hline 29 & $e$ & $\rho \mathrm{TMA}$-TMA rock \\
\hline 30 & $e$ & $\rho \mathrm{BX}_{3}-\mathrm{BX}_{3}$ rock \\
\hline
\end{tabular}

Table 5. Assignments of the skeletal fundamental frequencies of trimethylamine-boron halide complexes (in $\mathbf{~}^{-1}$ )

\begin{tabular}{|c|c|c|c|c|c|c|c|}
\hline \multirow[b]{2}{*}{ Fundamental } & \multicolumn{2}{|c|}{ Boron trifluoride } & \multicolumn{2}{|c|}{ Boron trichloride } & \multicolumn{2}{|c|}{ Boron tribromide } & \multirow{2}{*}{$\begin{array}{l}\text { Approximate } \\
\text { description }\end{array}$} \\
\hline & $\mathrm{B}^{11}$ & $\mathbf{B}^{10}$ & $\mathbf{B}^{11}$ & $\mathrm{~B}^{10}$ & $\mathrm{~B}^{11}$ & $\mathrm{~B}^{10}$ & \\
\hline \multicolumn{8}{|l|}{$A_{1}$ Class } \\
\hline$v_{6}$ & 842 & 847 & 833 & 835 & 827 & 830 & $\mathrm{C}-\mathrm{N}$ sireteh \\
\hline$y_{7}$ & 692 & 696 & 746 & 770 & 689 & 720 & $\mathrm{~B}-\mathbf{N}$ stretch \\
\hline$v_{8}$ & 932 & 952 & 538 & 540 & 518 & 520 & $\mathrm{~B}-\mathrm{X}$ stretch \\
\hline$v_{9}$ & 320 & 323 & 337 & 337 & 316 & 316 & $\mathrm{NC}_{3}$ deformation \\
\hline$v_{10}$ & 547 & $\mathbf{5 5 0}$ & 381 & 381 & 275 & 275 & $\mathrm{BX}_{3}$ deformation \\
\hline \multicolumn{8}{|l|}{$E$ Class } \\
\hline$v_{24}$ & 989 & 989 & 965 & 965 & 958 & 958 & $\mathrm{C}-\mathrm{N}$ stretch \\
\hline$v_{25}^{24}$ & 1142 & 1165 & 755 & 783 & 677 & 706 & B $\mathrm{X}$ stretch \\
\hline$v_{26}$ & 430 & 431 & 443 & 443 & 434 & 434 & $\mathrm{NC}_{3}$ deformation \\
\hline$v_{27}$ & 340 & 340 & 270 & 270 & 194 & 195 & $\mathrm{BX}_{3}$ deformation \\
\hline$v_{29}$ & $\cdots$ & $\cdots$ & 183 & 183 & 183 & $18: 3$ & $\mathrm{NC}_{3}$ rock \\
\hline$v_{30}$ & -- & - & -- & & - & - - & $\mathrm{BX}_{3}$ rock \\
\hline
\end{tabular}


GREENwood [24]. In addition, frequencies and assignments for the complex, $\mathrm{H}_{3} \mathrm{NBF}_{3}$, are available $[21,25]$ and a spectroscopic study has been reported for the pyridine: $\mathrm{BF}_{3}$ complex [26].

Modes involving motions of fluorine atoms commonly show unusually intense infrared absorptions and correspondingly weak Raman intensities. Accordingly, the asymmetric B-F stretching fundamental is assigned to the broad, intense infrared band at $1142 \mathrm{~cm}^{-1}$ which exhibits a significant $\mathrm{B}^{10}$ isotope shift. This frequency was too weak to be observed in the Raman spectrum of the normal species although a very weak line was observed in the spectrum of the isotopic species. Its isotope sensitivity and its absence in the spectra of the other complexes confirm its identity.

The situation with respect to the symmetric B-F stretching mode is somewhat more complicated. Normal coordinate calculations on $\mathrm{H}_{3} \mathrm{NBF}_{3}[27]$ have shown that the $\mathrm{B}-\mathrm{F}$ and $\mathrm{B}--\mathrm{N}$ stretching motions are strongly mixed to give two modes better described as out-of-phase and inphase symmetric $\mathrm{NBF}_{3}$ stretchings. A similar situation undoubtedly exists in the TMA complex. Since the out-of-phase motion should have an appreciable dipole moment change and involve a significant motion of the boron atom, this fundamental is assigned to the strong infrared band at 932 $\mathrm{cm}^{-1}$ which shifts to $952 \mathrm{~cm}^{-1}$ on isotopic substitution. Its Raman intensity is low as might be expected. The corresponding in-phase or breathing mode, $\nu_{7}$, is assigned to the strong Raman band at $694 \mathrm{~cm}^{-1}$ which shows a negligible isotope effect.

In the B-F deformation region, a pair of weak lines having the typical appearance of a Fermi doublet is observed in the Raman spectrum of both isotopic species. The higher of the two at about $545 \mathrm{~cm}^{-1}$ is assigned as the symmetric $\mathrm{BF}_{3}$ deformation on the basis of its greater infrared intensity while the lower at about $512 \mathrm{~cm}^{-1}$, is probably the overtone of the unobserved $\mathrm{BF}_{3}$ rocking mode. The asymmetricdeformation, $v_{27}$, is assigned to a band at $340 \mathrm{~cm}^{-1}$ in good agreement with a corresponding fundamental of the $\mathrm{BF}_{4}-$ ion.

\section{$\mathrm{BCl}_{3}$ group frequencies}

Although the spectrum of free $\mathrm{BCl}_{3}$ is well known [20], that of the $\mathrm{BCl}_{4}{ }^{-}$ion is incomplete $[28,29]$. The missing frequencies can be estimated from those of the isoelectronic $\mathrm{CCl}_{4}$ molecule, however, and in addition, numerous data are available for molecules containing the $\mathrm{CCl}_{3}$ group [30]. As in the fluoride molecule, the asymmetric chlorine stretch, $v_{25}$, should be strong in the infrared and exhibit a significant isotope shift. The logical choice is the intense band at $755 \mathrm{~cm}^{-1}$ which shifts to 783 $\mathrm{cm}^{-1}$ in the $\mathrm{B}^{10}$ compound. The appearance of the spectrum in this region is somewhat confused by overlapping with $\nu_{7}$ and the presence of an overtone. However,

[24] N. N. GreEnwood, J. Chem. Soc. 3811 (1954).

[25] J. Goubeau and J. Mitschelen, Z. Phys. Chem. 14, 61 (1958).

[26] H. Luther, D. Mootz and F. Radwitz, J. Prakt. Chem. 27ry, 242 (1958).

[27] R. C. TAYlor, Boron-Nitrogen Chemistry, Ch. 6, Advances in Chemistry Series No. 42, The American (Chemical Society (1964).

[28] N. N. Greenwood and K. Wade, J. Chem. Soc. 1130 (1960).

[29] T. C. Waddington and F. Klanberg, J. Chem. Soc. 2339 (1960).

[30] Landolt-Bonnstein, 6, Auflage, I. Band, Atom- und Molekular Physik, 2. Teil, Molekeln I. 
the intensities are altered in the Raman effect and $\nu_{7}$ can be distinguished more clearly.

Two strong Raman bands at 535 and $383 \mathrm{~cm}^{-1}$ were observed which, on the basis of their intensity, appear to be the symmetric $\mathrm{BCl}_{3}$ stretching and deformation modes. Their positions agree well with assignments in molecules containing the $\mathrm{CCl}_{3}$ group; for example, in 1,1,1-trichloroethane the corresponding frequencies fall at 525 and $343 \mathrm{~cm}^{-1}$. The remaining bands in the low frequency region can be assigned less reliably and the evidence is not sufficient to allow an unequivocal decision among all possible choices. The assignment of the asymmetric $\mathrm{BCl}_{3}$ deformation to the Raman band at $271 \mathrm{~cm}^{-1}$ is made on the basis of its somewhat greater intensity and sharpness than the band at $256 \mathrm{~cm}^{-1}$.

\section{$\mathrm{BBr}_{3}$ group frequencies}

No reference data other than the assignments of the free acid were found for the $\mathrm{BBr}_{3}$ group although frequencies for a few organic compounds containing the $\mathrm{CBr}_{3}$ group are available. However, most assignments could be made in a fairly straightforward manner except for the bands observed in the region between 650 and 750 $\mathrm{cm}^{-1}$. The infrared spectrum of the natural compound showed an intense band at $677 \mathrm{~cm}^{-1}$ with progressively weaker bands occurring at 707,732 and $752 \mathrm{~cm}^{-1}$ (Fig. 1); the $\mathrm{B}^{10}$ enriched compound showed a very strong band at $706 \mathrm{~cm}^{-1}$ with a much weaker band at $751 \mathrm{~cm}^{-1}$. The Raman effect, however, exhibited only two moderately intense bands at 673 and $689 \mathrm{~cm}^{-1}$ and at 700 and $720 \mathrm{~cm}^{-1}$ in the spectra of both the natural and enriched complexes respectively. The high infrared intensity and significant isotope shift suggest that the infrared bands at 677 and 706 $\mathrm{cm}^{-1}$ arise from the asymmetric $\mathrm{B}-\mathrm{Br}$ stretching motion, $v_{25}$, in the two isotopic species. The latter frequency also appears in the spectrum of the natural compound because of the appreciable amount of $\mathrm{B}^{10}$ present. The Raman bands at 673 and 700 $\mathrm{cm}^{-1}$ of the respective species are assigned to the same fundamental, the small difference from the infrared values probably arising from the difference in state. The other fundamental expected in this region and likely to have appreciable intensity in the Raman effect is $v_{7}$, a breathing type of motion involving the $\mathrm{B}-\mathrm{N}$ bond. This mode is assigned to the remaining two Raman frequencies at 689 and $720 \mathrm{~cm}^{-1}$ in the two isotopic compounds. Failure to observe $v_{7}$ in the infrared spectrum is not unexpected in view of the nature of the vibration. The infrared band at $752 \mathrm{~cm}^{-1}$ can easily be disposed of as a combination but the remaining moderately intense band at $732 \mathrm{~cm}^{-1}$ in the spectrum of the natural compound remains somewhat of a puzzle. The closest combination to this band is $v_{8}+v_{27}=713 \mathrm{~cm}^{-1}$ which is appreciably different and lower in frequency. It is possible that the $732 \mathrm{~cm}^{-1}$ band is an overtone or combination involving an inactive frequency, perhaps enhanced by resonance, but if this is so, it is odd that it is completely absent from the spectrum of the $\mathrm{B}^{10}$ species.

The symmetric $\mathrm{B}-\mathrm{Br}$ stretch, $v_{8}$, is assigned to the band at $516 \mathrm{~cm}^{-1}$ which, at first sight, might seem much too high when compared to the symmetric stretch in free $\mathrm{BBr}_{3}$ at $280 \mathrm{~cm}^{-1}$. However, this is not a valid comparison since the boron atom does not move in the symmetric vibration of the planar free halide whereas its amplitude undoubtedly is appreciable in the symmetric vibration of the pyramidal 
halogen structure in the complex. In fact, the rather small difference in $v_{8}$ between the chloride and bromide complexes indicates that the principal contribution to the reduced mass in the two cases comes from the boron atom.

\section{Complex frequencies}

The formation of the dative bond converts six external degrees of freedom into vibrational modes which can be described qualitatively as a $\mathrm{B}-\mathrm{N}$ stretch, degenerate $\left(\mathrm{CH}_{3}\right)_{3} \mathrm{~N}$ and $\mathrm{BX}_{3}$ rocking modes and the torsion of the two ends around the molecular axis. Of these, the torsion is inactive and the rocking modes are likely to be so low in frequency that their observation or positive identification will be difficult. In the case of the $\mathrm{B}-\mathrm{N}$ stretch, a study of several isotopic varieties of $\mathrm{H}_{3} \mathrm{NBH}_{3}$ [31] has located the uncoupled dative bond frequency at about $750 \mathrm{~cm}^{-1}$. However coupling effects with other skeletal frequencies undoubtedly will be present in most molecules containing terminal atoms heavier than hydrogen and it is questionable whether the dative bond can be said to have a characteristic bond frequency in such cases [27]. The fundamental, $v_{7}$, which nominally would be the dative bond stretch, probably is better described as a breathing type of motion of the $\mathrm{NBX}_{3}$ skeleton as mentioned earlier. It is assigned at nearly the same frequency, 692 and $689 \mathrm{~cm}^{-1}$, in the fluoride and bromide, respectively, but is significantly higher and at approximately the position of the uncoupled $\mathrm{B}-\mathrm{N}$ frequency in the chloride. No conclusions regarding the dative bond itself appear justified in the absence of a normal coordinate analysis.

\section{Conclusions}

Although there are minor details in each of the spectra for which explanations are unsatisfactory or lacking, by and large the picture presented for the principal features of the spectra of the three complexes appears consistent and logical. Since some final assignments were arrived at only by considering trends in the series, the advantage of having data from several closely related molecules can scarcely be overemphasized. Identification of the rocking modes associated with the two halves of the complexes and the one active torsional mode could not be made and must await more satisfactory data for the low frequency region.

Those fundamentals primarily associated with the acid portion of the complex in general were observed to fall intermediate between the corresponding frequencies of the free acid and the observed or extrapolated frequencies of the $\mathrm{BX}_{4}-$ ion. Because of the marked change in the geometry of the acid accompanying complex formation together with the extensive coupling among the low frequency modes, it is difficult to draw conclusions from the frequencies themselves regarding changes in bonding in the acid half of the complex.

In the case of fundamentals associated with the amine half, frequency shifts are more significant since structural changes are less severe than in the case of the acid. Two important factors have been recognized [32] as effecting the strength of amine $\mathrm{N}-\mathrm{C}$ or $\mathrm{N}-\mathrm{H}$ bonds when complexes are formed. The first is an increase in the

[31] R. C. Taylor and C. L. Cluff, Nature, 182, 390 (1958).

[32] V. N. Filmonov and D. S. Bystrov, Optics and Spectroscopy, 12, 31 (1962). 
amount of $s$ character in the sigma bonds resulting from changes in hybridization concomitant with the formation of the dative bond. This has the effect of increasing the bond strength. The second is the polarizing effect of the acid group which tends to bind the electrons in the bonding orbitals more tightly around the nitrogen and reduce the electron density in the valence bonds. The latter appears to predominate in the present case, since both $\mathrm{C}-\mathrm{N}$ frequencies in the complexes are lower than in the free amine. Data in the literature for other complexes show similar effects [32].

Factors influencing the forces involved in angle deformations are more numerous and less well understood than those for bond stretching and the interpretation of frequency changes is correspondingly more difficult. Reference has already been made to the fact that the symmetric $\mathrm{NC}_{3}$ deformation has been assigned to a lower frequency than in the free amine, a direction contrary to that found in ammonia complexes. Structural data for the TMA: $\mathrm{BF}_{3}$ complex [8] indicate a rather large increase in the $\mathrm{CNC}$ angle from $108^{\circ}$ to $114^{\circ}$ upon complexation, a change which also suggests that the restoring forces in the CNC deformation have increased. Corresponding structural data for $\mathrm{NH}_{3}: \mathrm{BF}_{3}$ are not available but confirmation of the present $\mathrm{NC}_{3}$ deformation assignment by data from the deuterated amine appears desirable before any discussion is attempted.

Acknowledgement-The support of this work by the National Science Foundation is gratefully acknowledged. Purchase of the multilayer interference filters was aided by a grant from the Rackham School of Graduate Studies. 